\title{
Neutral current interactions in ep scattering with longitudinally polarised leptons at $\mathrm{H1}$
}

\author{
Vladimir Chekelian (Shekelyan)* \\ MPI for Physics, HI Collaboration \\ E-mail: shekeln@mail.desy.de
}

The inclusive deep inelastic cross sections are measured for the neutral current process, $e^{ \pm} p \rightarrow$ $e^{ \pm} X$, in interactions with longitudinally polarised lepton beams of HERA II at large negative fourmomentum transfer squared $Q^{2} \geq 150 \mathrm{GeV}^{2}$. The polarisation asymmetries of the neutral current $e^{-} p$ and $e^{+} p$ cross sections are presented. The cross sections are combined with previously published data from $\mathrm{H} 1$ to obtain the most precise unpolarised measurements. The lepton charge asymmetry of the unpolarised cross sections is used to extract the structure function $x F_{3}^{\gamma Z}$. The measurements are found to be in agreement with the Standard Model expectations.

XVIII International Workshop on Deep-Inelastic Scattering and Related Subjects April 19 -23, 2010

Convitto della Calza, Firenze, Italy

\footnotetext{
* Speaker.
} 


\section{Introduction}

At the ep collider HERA (1992-2007), electrons of $27.5 \mathrm{GeV}$ collided with protons of 920 $\mathrm{GeV}$. This corresponds to an $e p$ center of mass energy of $320 \mathrm{GeV}$. The maximum negative fourmomentum-transfer squared from the lepton to the proton, $Q^{2}$, accessible with this machine was as high as $100000 \mathrm{GeV}^{2}$. After the luminosity upgrade of the machine in the second phase of the HERA program (HERA II, 2003-2007), the accelerator routinely provided the longitudinal polarization of the lepton beam of about $40 \%$. To achieve that pairs of spin rotators were implemented in the beam-line on either side of the interaction regions to rotate transversely polarized leptons into longitudinally polarized states and back again. The transverse polarization of the lepton beam arises naturally through synchrotron radiation via the Sokolov-Ternov effect. The degree of polarization was continuously measured using two independent polarimeters.

In this paper measurements of the inclusive neutral current (NC) cross sections are presented for $e^{+} p$ and $e^{-} p$ deep inelastic scattering. Both data sets are further subdivided into samples of left handed and right handed longitudinal polarization, $P=\left(N_{R}-N_{L}\right) /\left(N_{R}+N_{L}\right)$, where $N_{R}\left(N_{L}\right)$ is the number of right (left) handed leptons in the beam. The corresponding data sets are termed the $R$ and $L$ data sets respectively. The corresponding luminosity and longitudinal lepton beam polarization is given in Table 1 .

\begin{tabular}{|c|c|c|}
\hline & $R$ & $L$ \\
\hline$e^{-} p$ & $\mathscr{L}=45.9 \mathrm{pb}^{-1}$ & $\mathscr{L}=103.2 \mathrm{pb}^{-1}$ \\
& $P=(+36.9 \pm 2.3) \%$ & $P=(-26.1 \pm 1.0) \%$ \\
\hline$e^{+} p$ & $\mathscr{L}=98.1 \mathrm{pb}^{-1}$ & $\mathscr{L}=81.9 \mathrm{pb}^{-1}$ \\
& $P=(+32.5 \pm 1.2) \%$ & $P=(-37.6 \pm 1.4) \%$ \\
\hline
\end{tabular}

Table 1: Integrated luminosity, $\mathscr{L}$, and luminosity weighted longitudinal polarization, $P$, for the data sets at HERA II.

The longitudinal polarization of the lepton beam was exploited in the analysis of the full statistics of the HERA II period. The prediction of the Standard Model (SM) related to the difference of the NC cross sections for leptons with different helicity states arising from the chiral structure of the neutral electroweak exchange are investigated. The lepton charge asymmetry of the NC cross sections was used to measure the structure function $x F_{3}$ using combination of the unpolarized cross section measurements by H1 from HERA I and HERA II.

\section{NC cross sections with longitudinally polarized leptons}

The neutral current deep inelastic scattering cross section, after correction for QED radiative effects, can be written as

$$
\frac{\mathrm{d}^{2} \sigma_{\mathrm{NC}}^{ \pm}}{\mathrm{dxdQ}^{2}}=\frac{2 \pi \alpha^{2}}{x Q^{4}}\left(Y_{+} \tilde{F}_{2} \mp Y_{-} x \tilde{F}_{3}-y^{2} \tilde{F}_{L}\right)
$$

The reduced cross section is defined by $\tilde{\sigma}^{ \pm}\left(x, Q^{2}\right) \equiv \frac{\mathrm{d}^{2} \sigma_{\mathrm{NC}}^{ \pm}}{\mathrm{dxd}^{2}} \frac{x Q^{4}}{2 \pi \alpha^{2}} \frac{1}{Y_{+}} \equiv \tilde{F}_{2} \mp \frac{Y_{-}}{Y_{+}} x \tilde{F}_{3}-\frac{y^{2}}{Y_{+}} \tilde{F}_{L}$. Here, $x$ is the Bjorken scaling variable and $y$ characterizes the inelasticity of the interaction. The helicity dependences are contained in $Y_{ \pm}=1 \pm\left(1-y^{2}\right)$. 
The generalized proton structure functions, $\tilde{F}_{2,3}$, occurring in Eq.(1), may be written as linear combinations of the hadronic structure functions $F_{2}, F_{2,3}^{\gamma Z}$, and $F_{2,3}^{Z}$, containing information on the QCD parton dynamics as well as the electroweak couplings of the quarks to the neutral vector bosons. The function $F_{2}$ is associated with pure photon exchange terms, $F_{2,3}^{\gamma Z}$ correspond to photon- $Z^{0}$ interference and $F_{2,3}^{Z}$ correspond to pure $Z^{0}$ exchange terms. In addition, the longitudinal structure function $\tilde{F}_{L}$ may be similarly decomposed. $\tilde{F}_{L}$ is important only at high $y$ and is expected to be negligible at large $x$ and $Q^{2}$.

The generalized proton structure functions depend on the lepton beam charge, on the lepton beam polarization $P$, on the mass of the $Z^{0}$-boson $M_{Z}$ and $\sin ^{2} \theta_{W}, \theta_{W}$ being the electroweak mixing angle (Weinberg angle):

$$
\begin{array}{cl}
\tilde{F}_{2}^{ \pm}=F_{2}+k\left(-v_{e} \mp P a_{e}\right) F_{2}^{\gamma Z}+ & k^{2}\left(v_{e}^{2}+a_{e}^{2} \pm 2 P v_{e} a_{e}\right) F_{2}^{Z}, \\
x \tilde{F}_{3}^{ \pm}=k\left(-a_{e} \mp P v_{e}\right) x F_{3}^{\gamma Z}+ & k^{2}\left(2 v_{e} a_{e} \pm P\left(v_{e}^{2}+a_{e}^{2}\right)\right) x F_{3}^{Z} .
\end{array}
$$

Here, $k\left(Q^{2}\right)$ determines the relative amount of $Z$ to $\gamma$ exchange with $k=\left(4 \sin ^{2} \theta_{W} \cos ^{2} \theta_{W}\right)^{-1} \frac{Q^{2}}{Q^{2}+M_{Z}^{2}}$. The quantities $v_{e}=-1 / 2+2 \sin ^{2} \theta_{W}$ and $a_{e}=-1 / 2$ are the vector and axial-vector couplings of the electron to the $Z$ boson.

At leading order in QCD the hadronic structure functions are related to linear combinations of sums and differences of the quark and anti-quark momentum distributions $x q\left(x, Q^{2}\right)$ and $x \bar{q}\left(x, Q^{2}\right)$ of the proton:

$$
\begin{gathered}
\left(F_{2}, F_{2}^{\gamma Z}, F_{2}^{Z}\right)=x \sum\left(e_{q}^{2}, 2 e_{q} v_{q}, v_{q}^{2}+a_{q}^{2}\right)(q+\bar{q}), \\
\left(x F_{3}^{\gamma Z}, x F_{3}^{Z}\right)=2 x \sum\left(e_{q} a_{q}, v_{q} a_{q}\right)(q-\bar{q}),
\end{gathered}
$$

where $v_{q}$ and $a_{q}$ are the vector and axial-vector couplings of the light quarks to the $Z$ boson, and $e_{q}$ is the charge of the quark of flavor $q$.

\section{Results}

$\mathrm{NC}$ events in the $\mathrm{H} 1$ detector are characterized by an isolated high transverse momentum lepton and a hadronic system opposite in azimuth to the scattered lepton. Such events are triggered mainly using information from the liquid argon calorimeter. The calorimeter has finely segmented geometry allowing the selection of localized energy deposits in the electromagnetic sections of the calorimeter. This selection is found to be $100 \%$ efficient for leptons above $11 \mathrm{GeV}$.

Candidate NC interactions are selected by requiring the scattered lepton energy $E_{e}^{\prime}>11 \mathrm{GeV}$, $Q^{2}>133 \mathrm{GeV}^{2}$ and a reconstructed primary vertex within $35 \mathrm{~cm}$ in $z^{1}$ of the nominal interaction point. Background from misidentified leptons is suppressed by requiring a charged track to be associated to the lepton candidate. Longitudinal energy-momentum conservation requires that $E-$ $P_{z}=2 E_{e}$ where $E_{e}$ is the lepton beam energy and $E$ and $P_{z}$ are the usual components of the hadronic final state four-vector. By requiring $E-P_{z}>35 \mathrm{GeV}$ the influence of radiative corrections arising from initial state bremsstrahlung is considerably reduced. This requirement further suppresses the

\footnotetext{
${ }^{1}$ The $\mathrm{H} 1$ coordinate system is defined such that the positive $z$ axis is in the direction of the outgoing proton beam.
} 

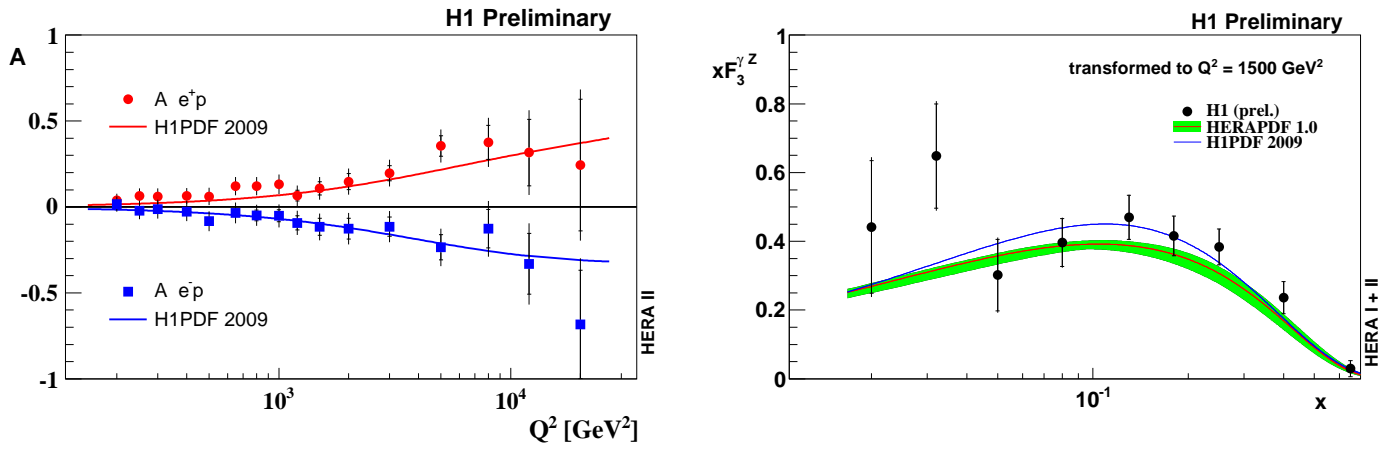

Figure 1: Left: The H1 measurement of the polarization asymmetry in the $e^{+} p$ and $e^{-} p$ NC interactions. Right: Measurements of the structure function $x F_{3}^{\gamma Z}$ by the H1 Collaboration. The curves describe the Standard Model predictions as obtained using PDFs from the NLO QCD fits to the unpolarized HERA I data.

contamination from photoproduction background in which a hadron is misidentified as a lepton candidate.

Using the four HERA II data set, see Table 11, the inclusive single differential cross section $d \sigma / d Q^{2}$ for $y<0.9$ and the reduced double differential cross section $\tilde{\sigma}^{ \pm}\left(x, Q^{2}\right)$ are measured [1] for the $e^{-} p$ and $e^{+} p$ scattering with the $L$ and $R$ polarization of the lepton beam. The cross section measurements extend over the $Q^{2}$ range 150 to $30000 \mathrm{GeV}^{2}$ and over the $x$ range 0.0032 to 0.65 .

In order to improve the statistical precision of the measurements the data from $L$ and $R e^{-} p$ $\left(e^{+} p\right)$ samples are combined together and corrected for the remaining residual polarization to provide a measurement of the unpolarized $e^{ \pm} p$ cross sections. They are combined with previously published H1 measurements [2, 3] from HERA I. These combined unpolarized cross sections make use of the complete HERA data set collected by $\mathrm{H} 1$ and amounts to a total luminosity of $165.5 \mathrm{pb}^{-1}$ for $e^{-} p$ scattering and $280.8 \mathrm{pb}^{-1}$ for $e^{+} p$ scattering.

\section{Polarization asymmetry}

The charge-dependent longitudinal polarization asymmetries of the NC cross sections are defined as

$$
A\left(e^{ \pm} p\right)=\frac{2}{P_{R}-P_{L}} \cdot \frac{\sigma^{ \pm}\left(P_{R}>0\right)-\sigma^{ \pm}\left(P_{L}<0\right)}{\sigma^{ \pm}\left(P_{R}>0\right)+\sigma^{ \pm}\left(P_{L}<0\right)} .
$$

They measure to a very good approximation the structure function ratio

$$
A\left(e^{ \pm} p\right) \simeq \mp k a_{e} \frac{F_{2}^{\gamma Z}}{F_{2}} .
$$

These asymmetries are proportional to the combinations $a_{e} v_{q}$ and thus provide a direct measure of parity violation. In the Standard Model $A\left(e^{+} p\right)$ is expected to be positive and about equal to $-A\left(e^{-} p\right)$. At large $x$ the asymmetries measure the $d / u$ ratio of the valence quark distributions according to

$$
A\left(e^{ \pm} p\right) \simeq \pm k \frac{1+d_{v} / u_{v}}{4+d_{v} / u_{v}}
$$


The results for polarization asymmetries are shown in Fig. 1 (left). The polarization effects in the NC process are reliably established at large values of $Q^{2}$. The asymmetries are well described by the Standard Model predictions based on the H1PDF 2009 [3] and HERAPDF1.0 [ [ 4 ] QCD fits to the unpolarized HERA I data.

\section{Charge asymmetry and $x F_{3}$}

A variation of the lepton beam charge allows the structure function $x \tilde{F}_{3}$ to be measured using the combined unpolarized data from HERA I and HERA II. The structure function $x \tilde{F}_{3}$ can be obtained from the cross section difference between electron and positron data

$$
x \tilde{F}_{3}=\frac{Y_{+}}{2 Y_{-}}\left[\tilde{\sigma}^{-}\left(x, Q^{2}\right)-\tilde{\sigma}^{+}\left(x, Q^{2}\right)\right] .
$$

The dominant contribution to $x F_{3}$ arises from the $\gamma Z$ interference, which allows $x F_{3}^{\gamma Z}$ to be extracted according to $x F_{3}^{\gamma Z} \simeq-x \tilde{F}_{3} / k a_{e}$ by neglecting the pure $Z$ exchange contribution, which is suppressed by the small vector coupling $v_{e}$. The structure function $x_{3}^{\gamma Z}$ is non-singlet and has little dependence on $Q^{2}$. The measured $x F_{3}^{\gamma Z}$ at different $Q^{2}$ values can thus be averaged taking into account the small $Q^{2}$ dependence. The averaged $x F_{3}^{\gamma Z}$, determined for a $Q^{2}$ value of $1500 \mathrm{GeV}^{2}$, is shown in Fig.1 (right). The $x F_{3}^{\gamma Z}$ measurement, directly sensitive to the valence quark distributions, is well described by the NLO QCD fits [3, 4] to previously published unpolarized HERA I data.

\section{Summary}

The NC $e^{ \pm} p$ cross sections are measured using the HERA II data with the longitudinally polarized lepton beams. The polarization asymmetry of the cross sections is obtained and found to be consistent with the predicted behavior, demonstrating the parity violation at very small distances down to about $10^{-18} \mathrm{~m}$. The measurements are combined with earlier published $\mathrm{H} 1$ results yielding the unpolarized NC cross section measurements by H1 using the entire HERA data. The lepton beam charge dependence due to the interference between the photon and the $Z$ exchange is observed and used to measure the interference structure function $x F_{3}^{\gamma Z}$. The $x F_{3}^{\gamma Z}$ data add to the knowledge of the valence quark distributions at low Bjorken- $x$ and are in a good agreement with the expectation of the Standard Model.

\section{References}

[1] H1 Collaboration, H1prelim-09-042, http://www-h1.desy.de/publications/H1preliminary.short_list.html

[2] C. Adloff et al. [H1 Collaboration], Eur. Phys. J. C 13, 609 (2000);

C. Adloff et al. [H1 Collaboration], Eur. Phys. J. C 19, 269 (2001);

C. Adloff et al. [H1 Collaboration], Eur. Phys. J. C 30 (2003) 1;

F. D. Aaron et al. [H1 Collaboration], Eur. Phys. J. C 63 (2009) 625.

[3] F. D. Aaron et al. [H1 Collaboration], Eur. Phys. J. C 64 (2009) 561.

[4] F. D. Aaron et al. [H1 and ZEUS Collaborations], JHEP01 (2010) 109. 